\title{
Evaluation of ceftriaxone-sulbactam-disodium edetate adjuvant combination against multi-drug resistant Gram-negative organisms
}

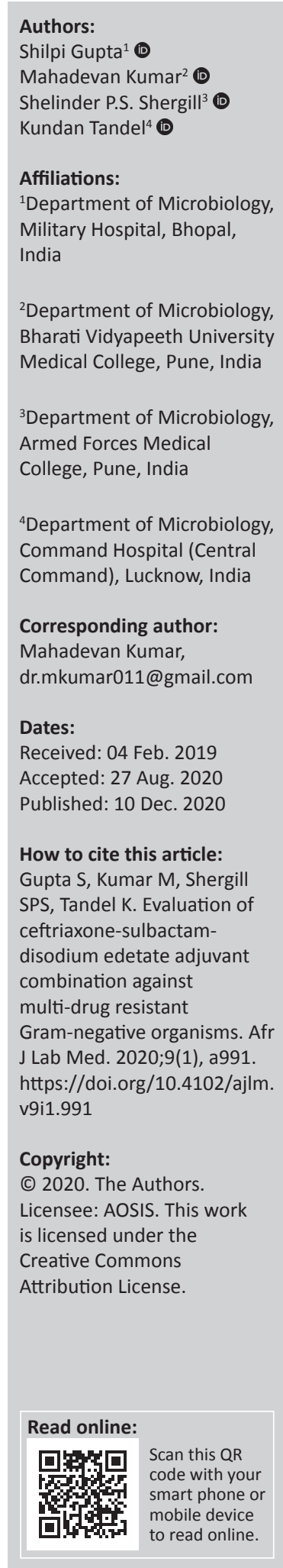

Background: Multi-drug resistant (MDR) Gram-negative bacteria are an emerging threat, both in hospital and community settings. As very few antibiotics are effective against such infections, the need of the hour is a new antibiotic or drug combination which can overcome the effect of extended-spectrum $\beta$-lactamases (ESBL) and metallo $\beta$-lactamases (MBL). A new antibiotic combination of ceftriaxone, sulbactam and disodium edetate (CSE) has recently been proposed to tackle the MDR organisms.

Objective: Our study was carried out to assess the susceptibility of ESBL- and MBL-producing Gram-negative organisms to CSE.

Methods: The study was conducted in a tertiary-care hospital in Delhi, India, from February 2017 to June 2017. A total of 179 MDR (85 ESBL + 94 MBL) Gram-negative isolates from various clinical samples, identified by an automated system (Vitek 2) were tested against CSE using the Kirby-Bauer disc diffusion method. Susceptibility to CSE was recorded based on interpretative zone sizes of ceftriaxone as per 2017 Clinical and Laboratory Standards Institute guidelines.

Results: The most common isolate was Escherichia coli $(76 / 179 ; 42.4 \%)$ followed by Klebsiella pneumoniae $(53 / 179 ; 29.6 \%)$ and Acinetobacter baumanii $(27 / 179 ; 15.1 \%)$. The in vitro susceptibility of ESBL- and MBL-producing Gram-negative isolates to CSE was found to be 58/85 (68.2\%) for ESBL and 37/94 (39.4\%) for MBL.

Conclusion: The in vitro susceptibility results obtained for CSE against ESBL-producing organisms is promising. It has the potential to emerge as a carbapenem-sparing antibiotic, active against ESBL-producing strains. Further clinical studies are required to establish the clinical efficacy of CSE against MDR pathogens.

Keywords: ceftriaxone sulbactam, disodium edetate; multi-drug resistance; carbapenemsparing.

\section{Introduction}

Increased antimicrobial resistance of Gram-negative bacteria (GNB), both hospital- and community-acquired, is of great concern worldwide. ${ }^{1}$ According to the report of Global Antibiotic Resistance Partnership - India Working Group, the irrational and increased use of antibiotics, especially cephalosporins, in India has resulted in the emergence of multi-drug resistant (MDR) bacteria which were earlier known to be susceptible. ${ }^{2}$ The menace of the emerging threat has been realised by the Indian Government, hence it has called for effective action to address the increasing antimicrobial resistance. The Indian Ministry of Health and Family Welfare, in collaboration with the World Health Organization, formulated the National Health Policy 2017, which calls for an urgent need for standardisation of antibiotic usage guidelines to minimise the emergence of antimicrobial resistance. Thus, both organisations decided to tackle the issue as a priority collaborative work in 2018-2019. ${ }^{3}$

Despite increasing antibiotic resistance, the common therapy for treatment of GNB infections includes use of $\beta$-lactams with $\beta$-lactamase inhibitors, third and fourth generation cephalosporins and carbapenems. ${ }^{4}$ Production of $\beta$-lactamase enzymes is the most widespread mechanism of resistance adopted by GNB to counteract the effect of $\beta$-lactam antibiotics. ${ }^{5,6}$ Extended-spectrum $\beta$-lactamases (ESBLs) are usually plasmid-mediated $\beta$-lactamases and hydrolyse oxyimino groupcontaining $\beta$-lactam antibiotics. ${ }^{7}$ Metallo $\beta$-lactamases (MBLs) are a class of broad-substrate 
spectrum enzymes that also hydrolyse most $\beta$-lactam antibiotics, except monobactams. ${ }^{8}$ Other mechanisms that contribute to resistance are drug efflux systems, outer membrane protein changes, antibiotic-modifying enzymes and antibiotic-target modification. ${ }^{9}$ Carbapenems are used against the ESBL-producing organisms because of their stability against hydrolysis by ESBLs and broad-spectrum activity. ${ }^{10}$ However, with the emergence of carbapenemhydrolysing enzymes, overexpression of efflux pumps and changes in outer membrane proteins, increases in carbapenem resistance have been reported among the members of enterobacteriaceae and non-fermenter GNB, such as the Acinetobacter and Pseudomonas group of pathogens. ${ }^{11,12}$

In India, the prevalence of ESBL and MBL producers among Gram-negative organisms range between $28 \%-84 \%$ and $7.5 \%-71 \%$, respectively. ${ }^{13,14}$ The increasing resistance towards available antibiotics, as well as the lack of development of new antibiotics against GNB, could soon lead to the world experiencing the tough situations of the pre-antibiotic era with an increase in cases with untreatable infections. A newer approach to improving the efficiency of the existing antimicrobials is the use of antibiotic adjuvants. A novel antibiotic adjuvant entity of ceftriaxone, sulbactam with adjuvant disodium edetate (CSE) is being used in Indian hospitals against MDR infections. ${ }^{15,16}$ The antibiotic adjuvant entity is a combination of ceftriaxone plus sulbactam with disodium edetate and has undergone Phase III clinical trials under the aegis of the Central Drugs Standard Control Organisation, India. ${ }^{17}$ This study aimed to study the in vitro susceptibility to CSE of MDR Gram-negative organisms isolated in our centre. Thus, the present study aimed to study the in vitro susceptibility of ESBL- and MBL-producing GNB to CSE and to explore whether it could be utilised as a carbapenem-sparing drug.

\section{Methods}

\section{Ethical considerations}

Ethical clearance was obtained from the Institutional Ethics Committee, Army Hospital (Research and Referral), New Delhi, India (92/2016)

\section{Study design and setting}

This cross-sectional study was conducted in the Department of Microbiology of a 900-bed, tertiary-care, super-speciality Army Hospital (Research and Referral), New Delhi, India from February 2017 to June 2017. Isolates were obtained from various clinical samples from both out- and inpatients received in the laboratory for bacterial culture from different clinical departments. Sample types included: urine, pus, cerebrospinal fluid, sputum, tissue from burn wound sites, endotracheal aspirate, semen, high vaginal swab and drains fluid.

\section{Microbiological processing}

Samples were processed using conventional methods. Blood culture bottles were incubated in a fully automated blood culture system, the BacT/Alert 3D (bioMérieux SA, Marcyl'Étoile, France). After obtaining a pure bacterial growth, isolate identification and antibiotic sensitivity testing were carried out on a Vitek 2 Compact (bioMérieux SA, Marcy-l'Étoile, France). An MDR isolate was defined as a GNB strain that showed resistance to at least three different categories of antibiotics. ${ }^{18}$ A total of 85 ESBL- and 94 MBL-producing GNB were identified by phenotypic tests and confirmed by the Vitek 2 system for inclusion in the study. The confirmatory test for ESBL production was carried out using discs containing ceftazidime (30 $\mu \mathrm{g})$ alone, along with ceftazidime/clavulanic acid $(30 / 10 \mu \mathrm{g})$ discs. Similarly, cefotaxime $(30 \mu \mathrm{g})$ and cefotaxime/clavulanic acid discs $(30 / 10 \mu \mathrm{g})$ were also used. An increase in zone diameter of $\geq 5 \mathrm{~mm}$ around the clavulanate disk compared to the zone of inhibition for the ceftazidime and cefotaxime disk alone was used to confirm and isolate as positive for ESBL production as per Clinical and Laboratory Standards Institute guidelines. ${ }^{19}$ The modified Hodge test ${ }^{19}$ was used for isolates identified as carbapenemase-producing GNB strains. A $10 \mu \mathrm{g}$ meropenem disc was placed on a Mueller-Hinton agar plate previously inoculated with Escherichia coli American Type Culture Collection 25922 (the indicator organism). Afterwards, the test organisms were streaked out in a straight line, starting from the edge of the meropenem disc, for at least $20 \mathrm{~mm}-25 \mathrm{~mm}$ length. The enhancement of growth of the indicator organism along the test organism's streak line and zone of inhibition of the disk to form a cloverleaf appearance was considered as a positive indicator for carbapenemase production as per Clinical and Laboratory Standards Institute guidelines. ${ }^{19}$ For detection of class B carbapenemases (MBL), the doubledisc synergy test using imipenem and imipenem plus ethylenediaminetetraacetic acid disc was done. An organism with a zone size difference of $7 \mathrm{~mm}$ between imipenem and imipenem plus ethylenediaminetetraacetic acid discs was considered to be an MBL-producing strain. ${ }^{20}$

All 179 isolates were then further tested for antimicrobial susceptibility against CSE (Venus Medicine Research Centre, Baddi, Himachal Pradesh, India) by the Kirby-Bauer disc diffusion method on a Mueller-Hinton agar kept at $37^{\circ} \mathrm{C}$ for $16 \mathrm{~h}-18 \mathrm{~h}$ (Figure 1). Quality control of CSE antibiotic discs

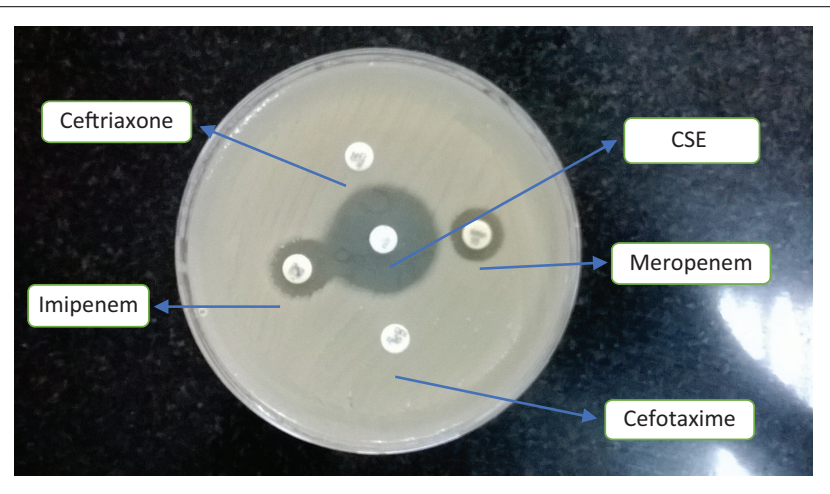

Note: Image shows representative zone of inhibition around ceftriaxone-sulbactamdisodium edetate in Mueller-Hinton agar in an organism resistant to other antibiotics at Army Hospital (Research and Referral), Delhi.

CSE, ceftriaxone-sulbactam-disodium edetate.

FIGURE 1: Zone of inhibition around the ceftriaxone-sulbactam-disodium edetate disc, Delhi, India, February 2017 to June 2017. 
was done using E. coli American Type Culture Collection 25922 and a laboratory-characterised sensitive isolate of Acinetobacter baumanii (Strain no. AHRR1205/2017). Susceptibility of the tested organisms against this combination was reported as sensitive, intermediate or resistant based on the zone of inhibition mentioned for ceftriaxone as per Clinical and Laboratory Standards Institute guideline M100S27: Performance Standards for Antimicrobial Sensitivity Testing, 2017. ${ }^{19}$ The zone of inhibition around the disc was measured, and the organism was labelled as sensitive if the zone measured $>23 \mathrm{~mm}$ for enterobacteriaceae or $>21 \mathrm{~mm}$ for Acinetobacter, intermediate if $20 \mathrm{~mm}-22 \mathrm{~mm}$ (enterobacteriaceae) or $14 \mathrm{~mm}-20 \mathrm{~mm}$ (Acinetobacter), or resistant if $<19 \mathrm{~mm}$ for enterobacteriaceae or $<13 \mathrm{~mm}$ for Acinetobacter. ${ }^{19}$ Non-fermenters such as Pseudomonas aeruginosa and Burkholderia cepacia were not tested against CSE as these are known to be inherently resistant to ceftriaxone and there are no testing standards mentioned in Clinical and Laboratory Standards Institute guidelines. ${ }^{19}$

\section{Data analysis}

Statistical analysis was done using Graph Pad, a free online software offering by founder Dr Harvey Motulsky (GraphPad Software, San Diego, California, United States). Associations between two factors were drawn through univariate logistic regression using the Fischer exact test. P-values of less than 0.05 were considered statistically significant.

\section{Results}

A total of 179 clinical isolates from 168 clinical cases (117 male patients and 51 female patients) were included in the study, with a mean patient age of 43.22 years (range: 4-85 years). Most isolates were from urine, followed by pus and blood specimens, and these accounted for 136 (75.9\%) of the total isolates (Table 1). Of the included pathogens, 127 (70.9\%) were isolated from inpatients, and $29(16.2 \%)$ were isolated from patients in intensive care units (Figure 2).

Among the isolated pathogens, E. coli $(n=76 ; 42.4 \%)$ was the most predominant followed by Klebsiella pneumoniae $(n=53$; $29.6 \%$ ) and $A$. baumanii $(n=27 ; 15 \%)$ (Table 1). Eighty-five
$(47.5 \%)$ of the tested isolates were ESBL producers and 94 (52.5\%) were MBL producers. Fifty-eight (68.23\%) of the ESBL producers (Table 2) and 37 (39.36\%) MBL producers (Table 3 ) showed in vitro sensitivity towards CSE.

Among the identified ESBL-producing GNB, 44 (73.3\%) E. coli and $7(53.8 \%)$ K. pneumoniae showed sensitivity towards CSE, while 51 (85\%) E. coli and 11 (84.6\%) K. pneumoniae showed sensitivity towards meropenem. The most common MBL-producing GNBs, K. pneumoniae, A. baumanii and E. coli, showed $27.5 \%(n=11), 48 \%(n=12)$ and $31.3 \%(n=5)$ sensitivity, respectively, towards CSE and 2.5\% $(n=1), 0 \%$ and $6.3 \%(n=1)$ sensitivity, respectively, towards meropenem (data not shown). A statistically significant association was found when susceptibility to meropenem and CSE were compared $(p<0.001)$ in ESBL-producing E. coli. However, no statistically significant associations were seen when the CSE susceptibility pattern was compared to meropenem susceptibility patterns for other ESBL- and MBL-producing organisms. Twelve (48\%) of the MBL-producing A. baumanii isolates which were resistant to meropenem showed susceptibility to CSE (data not shown). Multi-drug resistant

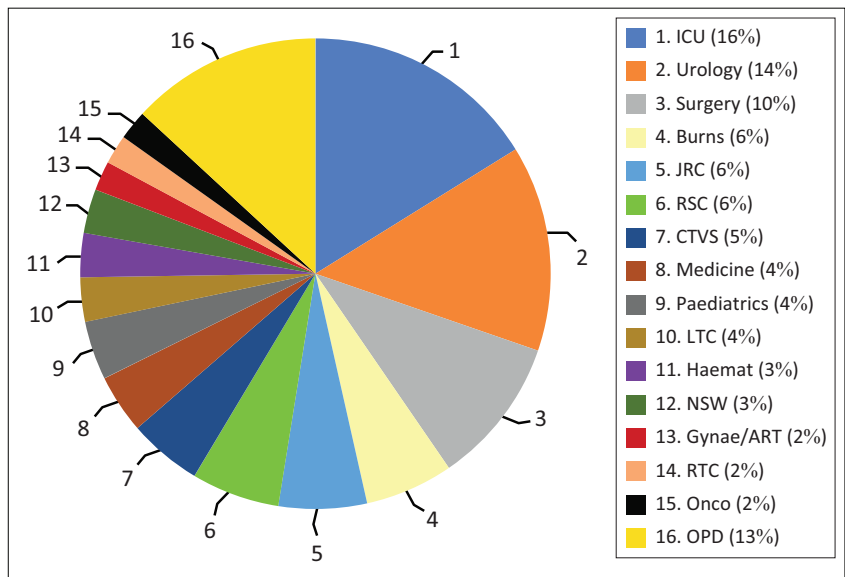

$N=179$

ICU, intensive care unit; JRC, Joint Replacement Centre; RSC, reconstructive surgery; CTVS, cardiothoracic and vascular surgery; LTC, Liver Transplant Centre; Haemat, Haematology; NSW, Neurosurgical ward; Gynae, gynaecology; ART, assisted reproductive technology; RTC Renal Transplant Centre; Onco, Oncology; OPD, out-patient department.

FIGURE 2: Ward distribution of samples included in the study at a hospital in Delhi, India, February 2017 to June 2017.

TABLE 1: Prevalence of individual pathogens in various samples at a hospital in Delhi, India, February 2017 to June 2017.

\begin{tabular}{|c|c|c|c|c|c|c|c|c|c|}
\hline \multirow[t]{2}{*}{ Sample } & \multirow{2}{*}{$\begin{array}{c}\text { Total no. of } \\
\text { isolates }(n=179)\end{array}$} & \multicolumn{8}{|c|}{ Isolates } \\
\hline & & $\begin{array}{l}\text { Escherichia coli } \\
\qquad(n=76)\end{array}$ & $\begin{array}{c}\text { Klebsiella } \\
\text { pneumoniae } \\
(n=53)\end{array}$ & $\begin{array}{c}\text { Acinetobacter } \\
\text { baumanii } \\
(n=27)\end{array}$ & $\begin{array}{c}\text { Proteus } \\
\text { mirabilis } \\
(n=13)\end{array}$ & $\begin{array}{c}\text { Enterobacter } \\
\text { cloacae } \\
(n=7)\end{array}$ & $\begin{array}{c}\text { Citrobacter } \\
\text { freundii } \\
(n=1)\end{array}$ & $\begin{array}{c}\text { Serratia } \\
\text { marcescens } \\
(n=1)\end{array}$ & $\begin{array}{c}\text { Morgenella } \\
\text { morganii } \\
(n=1)\end{array}$ \\
\hline Urine & 84 & 49 & 22 & 2 & 8 & 1 & 1 & 1 & 0 \\
\hline Pus & 34 & 10 & 5 & 12 & 4 & 3 & 0 & 0 & 0 \\
\hline Blood & 18 & 6 & 10 & 2 & 0 & 0 & 0 & 0 & 0 \\
\hline Tracheal aspirate & 11 & 1 & 2 & 7 & 0 & 1 & 0 & 0 & 0 \\
\hline Sputum & 6 & 1 & 3 & 2 & 0 & 0 & 0 & 0 & 0 \\
\hline CSF & 5 & 1 & 4 & 0 & 0 & 0 & 0 & 0 & 0 \\
\hline Drain fluid & 4 & 2 & 2 & 0 & 0 & 0 & 0 & 0 & 0 \\
\hline Semen & 4 & 2 & 1 & 0 & 0 & 1 & 0 & 0 & 0 \\
\hline High vaginal swab & 2 & 1 & 1 & 0 & 0 & 0 & 0 & 0 & 0 \\
\hline Total & 179 & 76 & 53 & 27 & 13 & 7 & 1 & 1 & 1 \\
\hline
\end{tabular}

CSF, cerebrospinal fluid 
TABLE 2: Antibiogram for ceftriaxone-sulbactam-disodium edetate against extended-spectrum $\beta$-lactamases producing Gram-negative isolates at a hospital in Delhi, India, February 2017 to June 2017.

\begin{tabular}{|c|c|c|c|c|c|c|c|c|}
\hline \multirow[t]{3}{*}{ Organism } & \multirow{2}{*}{\multicolumn{2}{|c|}{ No. of isolates $(n=85)$}} & \multicolumn{6}{|c|}{ CSE } \\
\hline & & & \multicolumn{2}{|c|}{ Susceptible $(n=58)$} & \multicolumn{2}{|c|}{ Intermediate $(n=22)$} & \multicolumn{2}{|c|}{ Resistant $(n=5)$} \\
\hline & $n$ & $\%$ & $n$ & $\%$ & $n$ & $\%$ & $n$ & $\%$ \\
\hline Escherichia coli & 60 & 70.5 & 44 & 73.3 & 13 & 21.7 & 3 & 5.0 \\
\hline Klebsiella pneumoniae & 13 & 15.3 & 7 & 53.8 & 6 & 46.2 & 0 & - \\
\hline Acinetobacter baumanii complex & 2 & 2.4 & 1 & 50.0 & 1 & 50.0 & 0 & - \\
\hline Proteus mirabilis & 3 & 3.5 & 2 & 75.0 & 0 & - & 1 & 25.0 \\
\hline Enterobacter cloacae & 4 & 4.7 & 2 & 50.0 & 1 & 25.0 & 1 & 25.0 \\
\hline Citrobacter freundii & 1 & 1.2 & 0 & 0.0 & 1 & 100.0 & 0 & - \\
\hline Morganella morganii & 1 & 1.2 & 1 & 100.0 & 0 & - & 0 & - \\
\hline Serratia spp. & 1 & 1.2 & 1 & 100.0 & 0 & - & 0 & - \\
\hline
\end{tabular}

CSE, ceftriaxone, sulbactam and disodium edetate

TABLE 3: Antibiogram for ceftriaxone-sulbactam-disodium edetate against metallo $\beta$-lactamases producing Gram-negative isolates at a hospital in Delhi, India, February 2017 to June 2017.

\begin{tabular}{|c|c|c|c|c|c|c|c|c|}
\hline \multirow[t]{3}{*}{ Organism } & \multirow{2}{*}{\multicolumn{2}{|c|}{ No. of isolates $(n=94)$}} & \multicolumn{6}{|c|}{ CSE } \\
\hline & & & \multicolumn{2}{|c|}{ Susceptible $(n=37)$} & \multicolumn{2}{|c|}{ Intermediate $(n=36)$} & \multicolumn{2}{|c|}{ Resistant $(n=21)$} \\
\hline & $n$ & $\%$ & $n$ & $\%$ & $n$ & $\%$ & $n$ & $\%$ \\
\hline Escherichia coli & 16 & 17.0 & 5 & 31.3 & 7 & 43.8 & 4 & 6.7 \\
\hline Klebsiella pneumoniae & 40 & 42.6 & 11 & 27.5 & 17 & 42.5 & 12 & 30.0 \\
\hline Acinetobacter baumanii complex & 25 & 26.6 & 12 & 48.0 & 9 & 36.0 & 4 & 16.0 \\
\hline Proteus mirabilis & 10 & 10.6 & 8 & 80.0 & 2 & 20.0 & 0 & - \\
\hline Enterobacter cloacae & 3 & 3.2 & 1 & 33.3 & 1 & 33.3 & 1 & 33.3 \\
\hline
\end{tabular}

CSE, ceftriaxone, sulbactam and disodium edetate

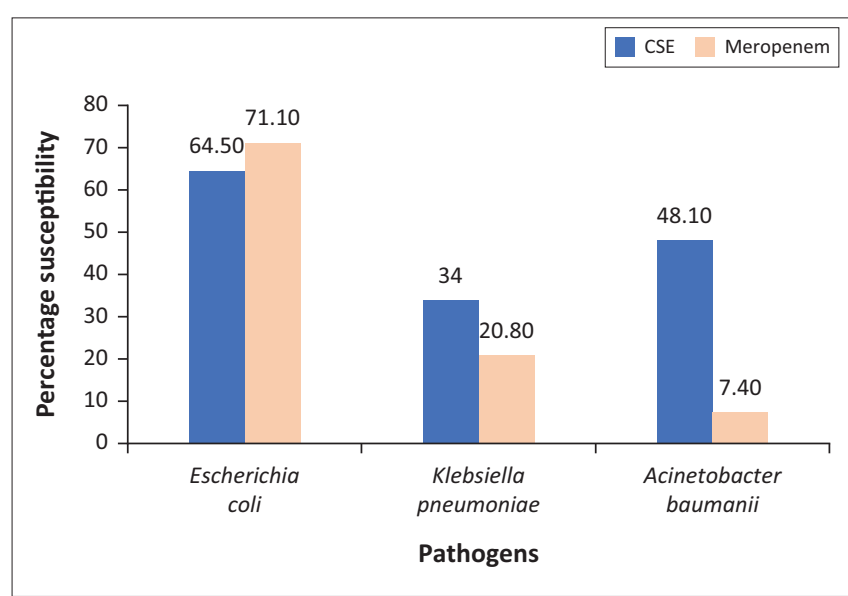

CSE, ceftriaxone-sulbactam-disodium edetate.

FIGURE 3: In vitro antibiotic susceptibility pattern for most common Gramnegative isolates against ceftriaxone-sulbactam-disodium edetate and Meropenem $(n=156)$ at a hospital in Delhi, India, February 2017 to June 2017.

E. coli, K. pneumoniae and A. baumanii showed $64.5 \%(n=49)$, $33.9 \%(n=18)$ and $48.1 \%(n=13)$ susceptibility, respectively, towards CSE and 71.1\% $(n=54), 20.8 \%(n=11)$ and $7.4 \%$ $(n=2)$, respectively, against meropenem (Figure 3$)$.

\section{Discussion}

This study included only those MDR GNB isolates which were proven to be pathogenic, obtained from clinically established cases of infection. The majority of isolates were from urine samples $(n=84 ; 47 \%)$, followed by pus $(n=34$; $19 \%)$ and blood $(n=18 ; 10 \%)$. The identified pathogens included E. coli, K. pneumoniae and A. baumanii, in decreasing order of prevalence. Similar distributions of isolates with similar specimen distribution have been reported by other studies. ${ }^{21,22}$ In the present study, E. coli $(n=49 ; 58.3 \%)$ was highly prevalent in urine samples, followed by K. pneumoniae ( $n=22 ; 26.2 \%)$, indicating their significant role in urinary tract infections. Similar findings were also reported by Janifer et al. in Chennai, South India ${ }^{23}$ and Ruchika et al. in Gurgaon, Haryana, North India. ${ }^{24}$ Klebsiella spp. and E. coli are known to be the major causative agents for hospitalacquired infections. According to the National Health Service report of 2017 on Gram-negative bloodstream infections, E. coli, P. aeruginosa and Klebsiella spp. were responsible for $72 \%$ of all Gram-negative bloodstream infections, with E.coli accounting for $59 \%$ of the total cases. ${ }^{25}$ However, in the present study, E. coli and K. pneumoniae were implicated in 16 out of $18(88.8 \%)$ Gram-negative bloodstream infection cases, with K. pneumoniae identified in the majority $(10 / 18 ; 55.5 \%)$ of cases as compared to E. coli $(6 / 18 ; 33.3 \%)$, which is in contrast to the National Health Service report. ${ }^{25}$ Acinetobacter baumanii is known to be an important pathogen in causing respiratory infections such as hospital-acquired pneumonia and bacteraemia, especially in intensive care patients, followed by skin and soft tissue infections and urinary tract infections. ${ }^{26,27}$ The present study has dissimilar findings in terms of isolation of A. baumanii, with maximum isolation from pus, because of skin and soft tissue infection cases $(50 \%)$; followed by respiratory samples, because of respiratory infections (32.1\%); and blood samples, because of bacteraemia $(7.1 \%)$.

In the present study, 95 out of 179 (53.1\%) MDR GNB isolates showed sensitivity to CSE in vitro. In similar studies conducted in the northern and western parts of India, higher susceptibility rates against CSE have been reported by Bhatia et al. $(84 \%-94 \%)$, Kumar et al. $(81.9 \%-94.74 \%)$, Bagga et al. $(87.5 \%-94.6 \%)$ and Sachdeva et al. $(74.2 \%-80.5 \%) .{ }^{12,24,28,29}$ 
The lower rate in our study compared to other studies could be because the isolates tested in the present study have been identified as ESBL or MBL producers, which was not specifically mentioned by other studies. The susceptibility of ESBL- and MBL-producing isolates to the CSE combination in the present study was from $68 \%$ for ESBL producers and $39 \%$ for MBL producers. The range is close to a similar study conducted in Mumbai, Maharashtra, India by Sahu et al. ${ }^{30}$

Because of increased clinical use of carbapenems against MDR GNB, our study also compared the efficacy of CSE against meropenem on Gram-negative isolates to use this new combination as a possible carbapenem-sparing drug. Carbapenems are considered to be the drug of choice for ESBL-producing GNB. The present study showed that 58 (68.2\%) of the ESBL isolates were susceptible to CSE, of which E. coli susceptibility to CSE was statistically significant when compared with its susceptibility to meropenem $(p<0.001)$. The susceptibility profile to CSE of the three most predominant pathogens in our study, E. coli $(49 / 76,64.5 \%)$, K. pneumoniae $(18 / 53,34 \%)$ and A. baumanii $(13 / 27,48.1 \%)$ was comparable for E.coli $(54 / 76,71.1 \%)$ when compared to meropenem and high for K. pneumoniae $(11 / 53,20.8 \%)$ and A. baumanii $(2 / 27$, $7.4 \%)$. However, several authors from different parts of India (Haryana, western Uttar Pradesh, Gujarat) have reported significantly higher susceptibility to CSE when compared with meropenem. ${ }^{24,28,31}$ Our study showed comparable sensitivity among ESBL organisms to CSE (58/85, 68.2\%) and meropenem $(64 / 85,75.3 \%)$, which implies that if CSE is tested against all ESBL isolates and they are found to be susceptible, CSE could be used as a drug of choice in place of carbapenems. Most of the MBL-producing organisms are resistant to carbapenems and the drug of choice for such isolates is polymixins. In the present study, 39.4\% (34) of such isolates were susceptible to CSE; thus, CSE instead of polymixins could be considered as a therapeutic option in these cases.

One major concern is finding effective treatments for infection with Acinetobacter spp., which is now commonly isolated from critical areas in most of the hospitals worldwide. ${ }^{24}$ Our study found CSE to be effective in $48.1 \%$ of MBL-producing A. baumanii infections, which is a fair number, and use of CSE could be beneficial in such infections. A similar study conducted in Pune, India found that CSE was a superior antibiotic compared to other commonly used $\beta$-lactam antibiotics, including carbapenems, when tested against MDR GNB. $^{32}$ A study conducted in Faridabad, Haryana, India, which evaluated the clinical use of CSE on patients, concluded that CSE should be used as a carbapenem-sparing drug and its combination with polymyxins can help to reduce mortality rates by successfully treating complicated MDR cases of intraabdominal, lower respiratory tract and urinary tract infections. ${ }^{12}$

\section{Limitations}

The limitation of our study was the relatively small number of isolates tested. Larger sample size and diverse health facility level (primary to tertiary) studies would be required to rule out any referral bias that is expected in a tertiary-care hospital. Further, this study can be extended with application to clinical situations to have a clinico-microbiological correlation to guide clinicians for the judicious use of CSE against MDR pathogens.

\section{Conclusion}

The results of this study show that CSE can potentially be effective among ESBL-producing bacteria, especially E. coli. The susceptibility of multi-drug resistant Gram-negative microorganisms to CSE suggests that CSE could be a good option as a carbapenem-sparing drug and also against some of the MBL-producing organisms.

\section{Acknowledgements}

We wish to thank Venus Remedies (India) for providing antibiotic discs of ceftriaxone-sulbactam-disodium edetate for testing.

\section{Competing interests}

There are no competing interests, financial interests, relationships and affiliations relevant to the subject matter or materials discussed in the manuscript.

\section{Authors' contributions}

All authors contributed intellectually to the work. S.G. was involved in bench work and manuscript writing; M.K. was involved in the study concept and supervision, manuscript writing and final approval; S.P.S.S. was involved in the study concept and supervision; and K.T. was responsible for collection of MDR strains, and contributed to the bench work.

\section{Sources of support}

This research received no specific grant from any funding agency in the public, commercial, or not-for-profit sectors.

\section{Data availability statement}

Data sharing is not applicable to this article as no new data were created or analysed in this study

\section{Disclaimer}

The views and opinions expressed in this article are those of the authors and do not necessarily reflect the official policy or position of any affiliated agency of the authors.

\section{References}

1. Kumar D, Singh AK, Ali MR, Chander Y. Antimicrobial susceptibility profile of extended spectrum ß-lactamase (ESBL) producing Escherichia coli from various clinical samples. Infect Dis (Auckl). 2014;7:1-8. https://doi.org/10.4137/IDRT. S13820

2. Global Antibiotic Resistance Partnership (GARP) - India Working Group. Rationalizing antibiotic use to limit antibiotic resistance in India. Indian J Med Res. 2011;134(3):281-294. 
3. National Action Plan on Antimicrobial Resistance (NAP-AMR) 2017-2021. April 2017 | Ministry of Health \& Family Welfare-Government of India.

4. Bush K. Bench-to-bedside review: The role of $\beta$-lactamases in antibiotic-resistant Gram-negative infections. Bush Crit Care. 2010;14:224. https://doi.org/10.1186/ cc8892

5. Bush K, Jacoby GA, Medeiros AA. A functional classification scheme for $\beta$ lactamases and its correlation with molecular structure. Antimicrob Agents Chemother. 1995;39(6):1211-1233. https://doi.org/10.1128/AAC.39.6.1211

6. Bush K, Jacoby GA. Updated functional classification of beta-lactamases. Antimicrobial Agents Chemother. 2010;54(3):969-976. https://doi.org/10.1128/ AAC.01009-09

7. Bradford PA. Extended-spectrum $\beta$-lactamases in the 21st century: Characterization, epidemiology, and detection of this important resistance threat Clin Microbiol Rev. 2001;14(4):933-951. https://doi.org/10.1128/CMR.14.4.933951.2001

8. Drawz SM, Bonomo RA. Three decades of $\beta$-lactamase inhibitors. Clin Microbiol Rev. 2010;23(4):160-201. https://doi.org/10.1128/CMR.00037-09

9. Chopra I, Schofield C, Everett M, et al. Treatment of health-care associated infections caused by Gram-negative bacteria: A consensus statement. Lancet Infect Dis. 2008;8(2):133-139. https://doi.org/10.1016/S1473-3099(08)70018-5

10. Pravin KN and Michelle SV. Prevalence of carbapenem resistant Enterobacteriaceae from a tertiary care hospital in Mumbai, India. J Microbiol Infect Dis. 2013;3(04):207-210. https://doi.org/10.5799/ahinjs.02.2013.04.0110

11. Rodrigues C. Carbapenem-resistant enterobacteriaceae: A reality check. Reg Health Forum. 2011;15(1):83-86.

12. Bhatia P. Alternative empiric therapy to carbapenems in management of drug resistant gram negative pathogens: a new way to spare carbapenems. Res J Infect Dis. 2015;3:2. http://doi.org/10.7243/2052-5958-3-2

13. Basavaraj CM, Jyothi P, Peerapur Basavaraj V. The prevalence of ESBL among Enterobacteriaceae in a tertiary care hospital of North Karnataka, India. J Clin Diagn Res. 2011;5(3):470-475.

14. Chaudhuri BN, Rodrigues C, Balaji V, et al. Incidence of ESBL producers amongst Gram-negative bacilli isolated from intra-abdominal infections across India (Based on SMART Study, 2007 Data). J Assoc Physicians Ind. 2011;59(5):287-292.

15. De AS, Kumar SH, Baveja SM. Prevalence of metallo- $\beta$-lactamase producing Pseudomonas aeruginosa and Acinetobacter species in intensive care areas in a tertiary care hospital. Indian J Crit Care Med. 2010;4(1):217-219. https://doi. org/10.4103/0972-5229.76089

16. Chaudhary $M$, Payasi A. Clinical, microbial efficacy and tolerability of Elores, a novel antibiotic adjuvant entity in ESBL producing pathogens: Prospective
randomized controlled clinical trial. J Pharmacy Res. 2013;7(4):275-280. https:// doi.org/10.1016/j.jopr.2013.04.017

17. Chaudhary M, Payasi A. A randomized, open label prospective, multicenter phaseIII clinical trial of Elores in lower respiratory tract and urinary tract infections. J Pharmacy Res. 2013;6(4):409-414. https://doi.org/10.1016/j.jopr.2013.04.011
18. Magiorakos AP, Srinivasan A, Carey RB, et al. Multidrug-resistant, extensively drug resistant and pandrug-resistant bacteria: An international expert proposal for interim standard definitions for acquired resistance. Clin Microbiol Infect. 2012;18(3):268-281. https://doi.org/10.1111/j.1469-0691.2011.03570.x

19. Clinical Laboratory Standard Institute (CLSI). Performance standards for antimicrobial susceptibility testing (2017) CLSI approved standards CLSI M100-S23. Wayne, PA: CLSI; 2017.

20. Clinical Laboratory Standard Institute. Performance standards for antimicrobial susceptibility testing; Twenty-second Informational Supplement. Wayne, PA Clinical Laboratory Standard Institute; 2013, p. 32.

21. AE Pop-Vicas, D'Agata EM. The rising influx of multidrug-resistant gram-negative bacilli into a tertiary care hospital. Clin Infect Dis. 2005;40(12):1792-1798. https://doi.org/10.1086/430314

22. Subhedar V, Jain SK. Gram negative super bugs: A new generation of ICU infections, an emerging challenge for health care settings. Am J Microbiol Res. 2016;4(1):47-50.

23. Janifer J, Geethalaksmi S, Satyavani K, et al. Prevalence of lower urinary tract infection in South Indian type 2 diabetic subjects. Indian J Nephrol. 2009;19(3):107-111. https://doi.org/10.4103/0971-4065.57107

24. Bagga R. Retrospective analysis of antibiotic susceptibility and resistance patterns against nosocomial Gram negative pathogens in Fortis Memorial Research Institute Gurgaon. Int J Curr Adv Res, 2015;4(9):347-351.

25. NHS_Improvement. Guidance on the definition of healthcare associated Gramnegative bloodstream infections. In: NHS Improvement, editor. Public Health England; 2017.

26. Gaynes R, Edwards JR. Overview of nosocomial infections caused by Gram-negative bacilli. Clin Infect Dis. 2005;41(6):848-854. https://doi.org/10.1086/432803

27. Paul M, Weinberger $M$, Siegman-Igra $Y$, et al. Acinetobacter baumannii: Emergence and spread in Israeli hospitals 1997-2002. J Hosp Infect. 2005;60(3):256-260. https://doi.org/10.1016/j.jhin.2005.01.007

28. Kumar M, Chaudhary S, Makkar DK, Garg N, Chugh SK. Comparative antimicrobial efficacy evaluation of a new product Elores against Meropenem on Gram-negative isolates. Asian J Pharm Clin Res. 2015;8:251-254.

29. Neelam Sachdeva. Antibiotic sensitivity pattern of bacterial pathogens in Rajeev Gandhi Cancer Hospital, Delhi. Int J Recent Sci Res. 2016;7(1):8480-8485.

30. Sahu M, Sanjith S, Bhalekar P, Keny D. Waging war against extended spectrum beta lactamase and metallobetalactamase producing pathogens - Novel adjuvant antimicrobial agent Cse1034-An Extended Hope. J Clin Diagn Res. 2014;8(6):DC20DC23. https://doi.org/10.7860/JCDR/2014/8802.4504

31. Makkar DK, Kumar M, Chaudhary S, Goyal S, Aggarwal P, Garg N. Comparative antimicrobial efficacy evaluation of a new product Elores against meropenem on Gram- negative isolates. Asian J Pharm Clin Res. 2015;8:1-4.

32. Arora S, Munshi N. Comparative assessment of antibiotic susceptibility pattern of Gram negative pathogens isolated from intensive care unit patients in Pune. BMRJ. 2015;10(2):1-9. https://doi.org/10.9734/BMRJ/2015/18199 